\title{
Depo-Provera (depot medroxyprogesterone acetate) use after bariatric surgery
}

\author{
Clarissa Lam' \\ Amitasrigowri S Murthy ${ }^{2,3}$ \\ 'New York University School of \\ Medicine, ${ }^{2}$ Department of Obstetrics \\ and Gynecology, Bellevue Hospital \\ Center, New York University \\ School of Medicine, ${ }^{3} \mathrm{New}$ York \\ University Langone Medical Center, \\ New York, NY, USA
}

This article was published in the following Dove Press journal:

Open Access Journal of Contraception

29 September 2016

Number of times this article has been viewed

\begin{abstract}
In the US, obesity rates are increasing greatly. The Centers for Disease Control and Prevention estimates that $68.5 \%$ of Americans, including $63.9 \%$ of adult women older than 20 years, are overweight (body mass index between $25 \mathrm{~kg} / \mathrm{m}^{2}$ and $29.9 \mathrm{~kg} / \mathrm{m}^{2}$ ) or obese (body mass index $>30 \mathrm{~kg} / \mathrm{m}^{2}$ ). In light of this, it is not surprising that the rates of bariatric surgery have also been increasing. When considering the metabolic changes associated with both bariatric surgery and contraceptive use, in combination with the unique medical considerations of obese women, it is indisputable that clear guidelines are needed when counseling obese patients of reproductive age after bariatric surgery. In this literature review, we focus on depot medroxyprogesterone acetate (DMPA) and the implications of its use in obese women, preweight and postweight loss following bariatric surgery. Both DMPA use and bariatric surgery are known to cause bone loss, but it is still unclear whether there is an additive effect of the two factors on bone loss and whether either of these factors directly leads to an increased risk of bone fracture. The current consensus guidelines do not impose a restriction on the use of DMPA after bariatric surgery. DMPA use is associated with weight gain, and it is unclear whether weight loss blunting occurs with the use of DMPA after bariatric surgery. Prior studies had demonstrated an association with weight gain in adolescents, and therefore, those prescribing DMPA use after bariatric surgery in adolescents should proceed with caution. Adult women do not have a similar response to the use of DMPA. DMPA use has rarely been associated with increased risk of venous thromboembolism (VTE). The obesity-associated increase in VTE should be mitigated by surgically induced weight loss. The concurrent use of DMPA in the post bariatric surgical period should not further increase the risk of VTE.
\end{abstract}

Keywords: bariatric surgery, DMPA, Depo-Provera, venous thromboembolism, bone loss, weight loss blunting, appetite changes

\section{Introduction}

Contraceptive counseling for reproductive-age women after bariatric surgery is still an area of ambiguity for obstetrician/gynecologists and bariatric surgeons alike. Although it is recommended that women avoid pregnancy for at least 12-18 months after surgery, no clear guidelines exist regarding the counseling of obese patients of reproductive age after bariatric surgery. By addressing this important issue of patient care, we hope to highlight an opportunity for coordination of care between bariatric surgeons and obstetrician/gynecologists caring for reproductive-age women pursuing surgical management of obesity. In this literature review, we take a closer look at the use of the injectable contraceptive, depot medroxyprogesterone acetate (DMPA).

Correspondence: Amitasrigowri S

Murthy

Department of Obstetrics and Gynecology, Bellevue Hospital Center, New York University School of Medicine, 550 First Avenue, NBV-9NI, New York, NY I0016, USA

Tel +I 2122630864

Email amitasrigowri.murthy@nyumc.org 
Approximately two-thirds of the world population lives in countries where deaths in those who are overweight or obese outnumber deaths in those who are underweight. ${ }^{1}$ In the US, the Centers for Disease Control and Prevention (CDC) estimates that $68.5 \%$ of Americans, including $63.9 \%$ of adult women older than 20 years, are overweight (body mass index [BMI] between $25 \mathrm{~kg} / \mathrm{m}^{2}$ and $29.9 \mathrm{~kg} / \mathrm{m}^{2}$ ) or obese (BMI $>30 \mathrm{~kg} / \mathrm{m}^{2}$ ). ${ }^{2}$ Because being overweight or obese is associated with a host of medical comorbidities, considerations in family planning are becoming increasingly complicated.

The importance of effective contraception is underscored by the unintended pregnancy rate. In the US in 2008, of the 6.6 million pregnancies, over half $(51 \%)$ were unintended ${ }^{1}$ and $43 \%$ of these resulted in abortion. The burden of unintended pregnancies is carried by women who are younger, poor, and unmarried; it is not surprising that these women also carry high rates of obesity. ${ }^{3}$

Reproductive-age women account for half of the patients undergoing bariatric surgical procedures in the US. ${ }^{4}$ Rapid weight loss after restrictive and malabsorptive bariatric surgical procedures usually improves fertility profiles, alters nutritional status, and may impact the efficacy of some contraceptive methods. Although the choice of optimal contraceptive methods for women after bariatric surgery is unclear, consensus guidelines, including those by the American College of Obstetricians and Gynecologists, recommend that pregnancy should be delayed for at least 12-18 months after surgery, to avoid the time of most rapid weight loss. ${ }^{5} \mathrm{~A}$ systematic literature review found that after bariatric surgery, maternal and fetal complications were decreased. ${ }^{6}$ In a survey of women in a postoperative bariatric surgery clinic at an academic medical center, $94.2 \%$ knew the importance of avoiding pregnancy for at least 1 year after surgery; yet, $16.3 \%$ were not using contraception. Only 4\% were using intrauterine devices (IUDs), and condoms were the most frequently used contraceptive method (30.6\%). ${ }^{7}$ Only $21.2 \%$ of patients had been referred to an obstetrician/gynecologist for contraceptive counseling and fertility planning. ${ }^{6}$ This discrepancy highlights an opportunity to improve coordination of care between bariatric surgeons and obstetrician/gynecologists caring for reproductive-age obese women undergoing surgical management of obesity.

Bariatric surgery has been shown to be the only intervention that results in consistent weight loss and demonstrates significant improvements in the comorbid conditions that exist in the obese population. This is in direct contrast to the rates of improvement after nonsurgical weight loss treatments. A large meta-analysis demonstrated that resolution of hypertension was observed in $68 \%$ of patients after Roux-en- $Y$ gastric bypass and only in $48 \%$ after adjustable gastric banding. ${ }^{8}$ Furthermore, as demonstrated in the Swedish Obese Subjects study - a matched cohort study performed over multiple centers across Sweden - all types of bariatric surgery were shown to be associated with more remissions of type 2 diabetes and fewer complications. ${ }^{9}$ The rates of both microvascular and macrovascular complications over 14 years were also decreased. ${ }^{9}$ Since 2011, the incidence of use of the various types of bariatric procedures for the treatment of obesity in the US has increased by $22 \%$ - with significant changes noted in the numbers of specific types of procedures. ${ }^{10}$ Currently, the most commonly performed procedure is the Roux-en-Y gastric bypass, followed by sleeve gastrectomy (SG) and adjustable gastric banding. ${ }^{10}$ Roux-en-Y gastric bypass compromises nearly half of all bariatric surgeries performed, and SG is increasing in popularity over gastric banding.

\section{Methods}

In order to perform a comprehensive literature review on the topic of DMPA use in obese women after bariatric surgery, we identified all published randomized controlled trials and cohort studies on four online databases (PubMed, MEDLINE, EMBASE, and Web of Science) by using relevant keywords to narrow down our search results. Then, the bibliographies of relevant articles ultimately included in our literature review were assessed for completeness. The search terms and the alternative search terms that were used are included in Table 1. Our search yielded 37 relevant articles to be included in the literature review.

\section{Efficacy and return to ovulation}

There have been many studies addressing the issue of hormonal contraceptive efficacy in overweight and obese women.

Table I Search terms and alternative search terms.

\begin{tabular}{lll}
\hline $\begin{array}{l}\text { Depo provera } \\
\text { (Depo-provera, } \\
\text { DepoProvera) }\end{array}$ & $\begin{array}{l}\text { Obesity } \\
\text { (Obese) }\end{array}$ & $\begin{array}{l}\text { Bariatric surgery } \\
\text { (bariatric surgical } \\
\text { procedure) }\end{array}$ \\
\hline $\begin{array}{l}\text { Medroxyprogesterone acetate } \\
\begin{array}{l}\text { Depo-medroxyprogesterone } \\
\text { acetate (Depo } \\
\text { medroxyprogesterone acetate) }\end{array}\end{array}$ & $\begin{array}{l}\text { Overweight } \\
\text { Morbid obesity }\end{array}$ & $\begin{array}{l}\text { Stomach stapling } \\
\text { Metabolic surgery }\end{array}$ \\
Cycrin & $\begin{array}{l}\text { Abdominal } \\
\text { obesity }\end{array}$ & Gastric bypass \\
Clinovir & & $\begin{array}{l}\text { Gastroplasty } \\
\text { Jejunoileal bypass } \\
\end{array}$ \\
& & Lipectomy \\
\hline
\end{tabular}


According to a 2009 literature review by Trussell et al, ${ }^{11}$ existing studies do not clearly demonstrate a cause-and-effect association between increased BMI and decreased contraceptive efficacy. This is similarly true for DMPA. Several studies have been able to demonstrate that BMI does not have an impact on DMPA efficacy. In a randomized, single-center trial conducted by Jain et al., it was found that there are no apparent differences in the pharmacokinetics of DMPA in overweight or obese women when compared to that in normal weight women. In addition, there is also no impact of BMI on the rate of return to ovulation after cessation of DMPA. The results of this study demonstrate that the effects of immediate and sustained ovulatory suppression of DMPA are observed regardless of the user's body weight. ${ }^{12}$ Similarly, the review article by Teal and Ginosar ${ }^{13}$ in 2007 demonstrated that the return to ovulation after a single dose of DMPA was not affected by the obesity index. Another study by Jain et $\mathrm{al}^{14}$ looking at the safety of DMPA also found that there was no attenuation of DMPA's contraceptive effects in women with a higher BMI.

\section{Bone loss}

Other metabolic changes can occur after bariatric surgery, and the choice of contraceptive method may either exacerbate or alleviate these conditions. One condition that is of concern is bone loss.

Obesity has been believed to be protective against osteoporosis due to higher bone mineral density (BMD). This increase in BMD is likely due to larger bone size, increased levels of estrogens due to aromatization of androgens from the adipose tissue, as well as changes in mechanical loading. ${ }^{15}$ However, evidence also demonstrates that obesity itself is a cause of altered levels of bone micronutrients and hormones. Vitamin D deficiency and elevated parathyroid hormone levels have been seen with higher BMI, thereby necessitating preoperative screening. In contrast to the metabolic improvements seen with diabetes and hypertension, bariatric surgery has been associated with bone loss.

The type of bariatric procedure determines the degree of both bone turnover and BMD loss. Long-term follow-up is recommended given the individual's risk of bone loss. Restrictive procedures have not been shown to affect BMD greatly. The changes seen are consistent with what occurs with weight loss alone. ${ }^{15}$ One study found no differences in body bone mineral content between that measured preoperatively before gastric banding and then again at 2 years postoperatively. ${ }^{16}$ In contrast to the minimal changes seen with gastric banding, gastric bypass often can result in calcium deficiency as well as increased bone turnover and decreased bone mass. Lower calcium levels are a direct result of poor absorption of calcium due to loss of the proximal bowel location of absorption as well as decreased dietary intake. ${ }^{17}$

Not much data exist on bone loss following the SG procedure. One study examined bone loss in patients who underwent a SG as compared to those who underwent a gastric bypass. Bone loss occurred in SG patients but at lower rates than in those after gastric bypass. ${ }^{18}$ Despite the increased rate of BMD loss and the altered hormone levels and decreased calcium following bariatric surgery, multiple studies have not supported an increased incidence in either osteoporosis or fracture. ${ }^{19}$ However, the accuracy of dual $\mathrm{X}$-ray absorptiometry (DXA) in evaluating BMD is in question. Measurements of the spine and hip are more difficult to both obtain and interpret than those of the radius or tibia. Many images are unreadable due to poor penetration of photons through soft tissue, as well as BMD artifact and changing body composition. ${ }^{20}$

Knowing about the effect of bone loss can affect the choice of contraceptive to use after bariatric surgery. DMPA is an injectable contraceptive commonly used across the world and has been available for use for $\sim 50$ years. It acts as a contraceptive by inhibiting gonadotropin secretion, thereby suppressing estradiol production and follicle formation. Given the hypoestrogenic state created, bone resorption increases while bone formation decreases; thereby resulting in loss of BMD. ${ }^{21}$ While the concern for bone loss is the highest among adolescent users of DMPA, only one study has actually evaluated BMD levels after starting and then discontinuing DMPA in adolescents. Scholes et al followed 170 adolescent girls using DMPA and measured BMD every 6 months. ${ }^{22}$ Among DMPA users, BMD declined at the hip and spine relative to nonusers. More rapid bone loss was seen in new DMPA users when compared to DMPA users of $>6$ months. ${ }^{22}$ Typical BMD loss with DMPA is noted to be $\sim 5 \%$ at both the femoral neck and the lumbar spine. This level of bone loss is similar to that seen with lactation. ${ }^{23}$ It is interesting, however, that the clinical significance of this decrease in BMD is unknown. What is known is that any decline in BMD noted with DMPA use is reversed after discontinuation, usually to levels seen at the time of initial DMPA start. ${ }^{24}$ Given this evidence, consensus statements from both the World Health Organization (WHO) and ACOG recommend that there be no restriction of use of DMPA in response to skeletal concerns. Furthermore, routine BMD testing is not recommended on the basis of this evidence. ${ }^{25,26}$ 
The question of whether or not DMPA can be used safely in reproductive-age women after undergoing bariatric surgery still requires clarification. In our opinion, given the lack of evidence that loss of BMD due to bariatric surgery is associated with increased fracture risk and that the use of DMPA leads to BMD loss and increased fracture risk, DMPA use should not be restricted in women who have undergone bariatric surgery. Whether monitoring of bone loss with DXA is necessary is still a question - routine monitoring in young women of reproductive age using DMPA is not recommended as DXA has not been validated in this population. In addition, given the inherent inaccuracy of DXA monitoring after bariatric surgery, it is our opinion that in those women being monitored with DXA, any noted bone loss would likely not correlate with an increased fracture risk. However, data on this question should be addressed with more long-term studies.

\section{Weight loss blunting}

Another concern after bariatric surgery is that of weight regain or weight loss blunting. It is unclear if the use of DMPA after bariatric surgery reduces the amount of weight lost. Another question is if the use of DMPA after bariatric surgery affects the amount of weight lost by changing a woman's appetite. Before looking at the effects of DMPA, how weight loss occurs after surgery needs to be clarified.

Although it has been proposed that malabsorption is one of the key mechanisms of the observed weight loss following a gastric bypass procedure, studies have not been able to attribute clinical significance to this effect. It has been demonstrated that the restrictive effects of bariatric surgery on the stomach volume play a crucial role in weight loss in gastric bypass patients. These restrictive effects lead to earlier satiety and the consumption of smaller meals.

This effect of appetite suppression, in particular, is proposed to be the dominant player in postoperative weight loss. Ghrelin is the hormone released in response to the physiological condition of an empty stomach, and as such, plays a crucial role in regulating feelings of hunger. In one groundbreaking study, ${ }^{25}$ it was found that ghrelin levels were significantly reduced in those who experienced weight loss after gastric bypass, thus suggesting that appetite was also significantly reduced after the surgical procedure. One study using the visual analog scale to assess appetite following gastric bypass versus SG found both surgeries to cause significant appetite-lowering effects. ${ }^{27}$ Although a decrease in food intake was noted in both groups, the gastric bypass group showed a gradual return in appetite, whereas the SG group was not observed to have the same return to baseline appetite levels. In fact, fasting and postprandial levels of ghrelin were noted to be significantly decreased. Multiple studies $^{8,9,10}$ examining both short-term and long-term outcomes of bariatric surgery have noted that while substantial weight loss is a clear outcome, there is significant variability in the amount of weight lost. The Swedish Obese Subjects study demonstrated a mean change in body weight of $23 \%$ in the first 2 years after surgery. ${ }^{28}$ This rate of weight lost was similarly seen in the Utah obesity studies. Six years following bariatric surgery, the patients were noted to have lost, on average, $27 \%$ of their body weight. ${ }^{29}$

Medroxyprogesterone acetate (MPA) is a synthetic progestin derived from 17oc- hydroxyprogesterone. During the initial evaluation of antitumor activity of this progestin, it was seen that the use of MPA was associated with an increase in weight and appetite. ${ }^{30,31}$ Reported weight gain is thought to be due to an increase in total body fat and not an increase in water retention. ${ }^{32}$ The proposed mechanism of MPA on weight and appetite is related to the in vitro production of serotonin and cytokines (interleukin [IL]-1, IL-6, and tumor necrosis factor alpha $[\mathrm{TNF}-\alpha]$ ) by peripheral blood mononuclear cells. ${ }^{31}$ Data from these studies have all used the oral formulation and not the depot format.

Specifically looking at the depot formulation, direct increase in adipose tissue is implicated in the weight gain observed. Furthermore, this increase in fat has been observed to accumulate in the body, primarily as visceral fat. The hypoestrogenic state created by DMPA use is the main explanation for DMPA-related increase in fat mass. ${ }^{33}$ This presumption is in agreement with the finding that in the hypoestrogenic state of menopause, observed increases in fat mass are independent of other factors such as age or baseline weight. ${ }^{33}$ However, studies have shown that this weight gain is not the result of increased food intake but rather the result of DMPA's regulatory effects on appetite and energy expenditure. It has also been postulated that another mechanism for the weight gain observed in DMPA users is a glucocorticoid-like effect on hormonal regulators of metabolism. However, the existing data have shown that DMPA-associated weight gain may be reversible. ${ }^{34}$

In those using the depot format, the significance of this weight gain is unclear. Evidence from five studies ${ }^{35}$ suggests that among adult women using DMPA, baseline weight or BMI is not associated with differences in weight gain. In a study of normal, overweight, and obese users of DMPA, adult obese women using DMPA did not gain more weight than control women using the copper IUD. ${ }^{36}$ However, the BMI 
in normal and overweight women using DMPA increased more rapidly than matched women using the copper IUD. ${ }^{37}$ In contrast, in a study comparing adolescent girls using DMPA to those using oral contraceptives or nonhormonal methods, weight gain in obese girls receiving DMPA was significantly greater than weight gain in obese girls receiving oral contraceptives $(P<0.001)$ and obese girls using no hormonal method $(P<0.001)$. Weight gain in obese girls receiving DMPA was also greater than weight gain in all nonobese categories $(P<0.001) .{ }^{32}$ In addition, a Cochrane review of progestin-only contraceptives demonstrated that the mean weight gain was low in the first year $-<2 \mathrm{~kg} .{ }^{37}$ Weight increases were noted in the 2 years following initiation of progestin-only contraceptives, but these were not significantly different across study groups. However, one study that followed women using DMPA for up to 10 years found that while women using both hormonal and nonhormonal methods of birth control gained weight, DMPA users were observed to have gained more weight $(6.6 \mathrm{~kg}$ in DMPA users, $4.9 \mathrm{~kg}$ in copper IUD users). ${ }^{38}$

In 2008, the WHO Expert Working Group reviewed the existing literature to evaluate the current medical eligibility criteria for the use of progestin-only contraceptives in obese women. The recommendations are that, in general, adolescents ( $<18$ years) with a BMI of $\geq 30 \mathrm{~kg} / \mathrm{m}^{2}$ can use DMPA (WHO Category 2) and other progestin-only methods (WHO Category 1). Obese adult women can use all progestin-only methods (WHO Category 1). The European Society of Contraception and Reproductive Health also found that DMPA was safe and effective in obese women.

Given that the rate of weight loss is the highest in the first year after bariatric surgery and that DMPA does seem to cause some weight gain, particularly in the first year of use, there may be some concerns for weight loss blunting with time since bariatric surgery. However, the minor weight gain observed in DMPA users is negligible when taking into consideration the rapidity of weight loss in the first year. It is, however, of concern that continued use of DMPA may cause a weight gain that may blunt the long-term success of bariatric surgery.

\section{Obesity and venous thromboembolism}

There is convincing epidemiologic evidence for an association between venous thromboembolism (VTE) and obesity from population studies. They demonstrate more than a twofold increase in the risk of VTE among obese patients. ${ }^{35}$ The risk of VTE is elevated fourfold to fivefold above baseline during pregnancy, but the increase in the risk of VTE specifically for obese pregnant women has been shown to be additive. Assuming an additive effect of obesity plus pregnancy on the risk of VTE, this risk may be clinically significant. One nested case-control study in Denmark demonstrated a fivefold increased risk of thromboembolism with obesity in early pregnancy. ${ }^{39}$ Prevention of undesired pregnancies in obese women is clearly a priority from the standpoint of reducing medical risks.

Proposed mechanisms for the association between obesity and VTE include physical effects and biochemical pathways. Visceral adipose tissue can cause increased delivery of free fatty acids to the liver. In addition to stimulation of production of coagulation proteins, free fatty acids can cause insulin resistance as well as oxidative stress ending in the triggering of systemic inflammation. This can enhance coagulation and decrease clot destruction, thus creating a prothrombotic state. ${ }^{40}$ This is consistent with the finding that higher waist circumference (a measure of abdominal/visceral fat) is associated with the highest risk of VTE in both men and women. In women, using hip circumference, a measure of subcutaneous fat, in addition to waist circumference was also associated with a higher risk of VTE. ${ }^{41}$ Thrombin formation has been demonstrated to be elevated in obese patients, and this may contribute to the overall prothrombotic state. Bariatric surgery and the following weight loss can cause a reduction in thrombin production. It is possible that with the surgically induced reduction in adipose tissue, free fatty acids, and thrombin, there may be a decrease in VTE risk after bariatric surgery. If exercise is added to bariatric surgery, then with the additional reduction of plasminogen activator inhibitor 1 as a result of regular activity, the decreased risk of VTE seen with weight loss may be further enhanced. ${ }^{42}$

Most progestins are not associated with an increase in VTE risk. As expected, DMPA has not been demonstrated to increase VTE risk. One meta-analysis examining the risk of VTE in progestin-only contraceptive users suggests a twofold increase in the risk of VTE with the use of DMPA. This is difficult to assess as the meta-analysis included only eight studies of which only two included DMPA users. ${ }^{43}$ In addition, there does not seem to be an additive increase in VTE risk in obese women using hormonal contraception, particularly in those using DMPA. DMPA has not been demonstrated to create a prothrombotic state. Therefore, the use of hormonal contraceptive methods remains an option for obese women. ${ }^{44}$ The Centers for Disease Control and Prevention medical eligibility criteria and the WHO list almost all hormonal methods as a Category 1 with combined hormonal methods 
being a Category $2 .{ }^{45}$ The European Society of Contraception and Reproductive Health also states that there is minimal evidence of an elevated VTE risk with DMPA. ${ }^{46}$ Hormonal contraceptive methods can be recommended provided that clinicians conduct a thorough discussion of risk of VTE and document it in the chart.

\section{Breast cancer risk}

Obesity is an established risk factor for the development of breast cancer. Some proposed mechanisms of this increased risk include disturbances in adipose tissue function, endocrine functions leading to increased estrogen, insulin resistance leading to hyperinsulinemia-induced activation of various cellular pathways, and the generalized increase in inflammatory cytokine levels. These changes promote proliferation of malignant cells and undermine the cell death pathways.$^{47}$ DMPA use has been implicated to play a role in the development of breast cancer. The results of the Women's Health Initiative's randomized controlled trials of postmenopausal hormone therapy comparing conjugated equine estrogen (CEE) alone and CEE plus MPA use demonstrated that breast cancer rates were considerably elevated following several years of CEE plus MPA use. This is in direct contrast to lower breast cancer rates following several years of CEEonly use. In this study, the authors state that an explanation for this difference is related to some unknown factor of MPA. ${ }^{48}$ Although it is difficult to assess the combined effects of obesity and DMPA on breast cancer risk, one paper ${ }^{49}$ noted the high proportion of overweight or obese women in both arms of the Women's Health Initiative study, perhaps explaining the effects of MPA and obesity on the rates of breast cancer. In a population-based case-control study in the US, the use of DMPA for $\geq 1$ year was associated with a twofold increased risk of breast cancer. ${ }^{50}$ This increase in risk does dissipate after cessation of use of DMPA. This study is complicated by the small numbers of cases and controls.

A different study highlighted by Byers and Sedjo showed that breast cancer risk was lowered by $83 \%$ after bariatric surgery and that the time to risk reduction was relatively short. This is believed to be mediated by the dramatic decrease in estradiol and increase in sex hormone binding globulin after intentional weight loss in women. In addition, the levels of inflammatory markers such as C-reactive protein, TNF- $\alpha$, and IL-6 all showed a marked decrease of approximately one-third after a seemingly trivial amount of weight loss. ${ }^{51}$

It remains unclear if long-term use of DMPA by women after bariatric surgery will increase the risk of development of breast cancer, particularly during the first year after surgery when weight change is the greatest.

\section{Conclusion}

There is still much to be evaluated when it comes to contraceptive counseling in this specific subset of reproductiveage women. The recommendation to avoid pregnancy for 12-18 months following bariatric surgery on the basis of avoiding the time of most rapid weight loss is clear but there are still crucial gaps in our knowledge of how to best counsel these women on contraceptive methods. DMPA is one method that has the potential to complicate various medical issues surrounding bariatric surgery in obese women, namely BMD loss, weight loss, and venous thromboembolic risk. In light of data from various studies presented above, however, we would feel comfortable recommending DMPA to most women of reproductive age after bariatric surgery. Considering the prior studies demonstrating that DMPA use in adolescents is associated with weight gain, those prescribing DMPA to adolescents after bariatric surgery should proceed with caution. In addition, the use of DMPA should be weighed against the risk of development of breast cancer. This literature review highlights the need for more long-term studies looking at these issues in order to significantly improve the care of reproductive-age obese women undergoing bariatric surgery.

\section{Disclosure}

Clarissa Lam has no conflicts of interest to disclose. Amitasrigowri S Murthy is a trainer for Nexplanon in Merck, a trainer for Mirena in Bayer, and a trainer for Liletta in Actavis. The authors report no other conflicts of interest in this work.

\section{References}

1. Finer LB, Zolna MR. Shifts in intended and unintended pregnancies in the United States, 2001-2008. Am J Public Health. 2014;104(suppl 1): S43-S48.

2. National Center for Health Statistics [webpage on the Internet]. Health, United States, 2011, with special feature on socioeconomic status and health [cited April 23, 2013]. Available from: http://www.cdc.gov/nchs/ data/hus/hus11.pdf\#069. Accessed May 2, 2016.

3. Finer LB, Zoina MR. Unintended pregnancy in the United States: incidence and disparities, 2006. Contraception. 2011;84(5):478-485.

4. Maggard MA, Yermilov I, Li Z, et al. Pregnancy and fertility following bariatric surgery: a systematic review. JAMA. 2008;300(19):2286-2296.

5. American College of Obstetricians and Gynecologists. ACOG committee opinion No. 549. Obesity in pregnancy. Obstet Gynecol. 2013;121(1):213-217.

6. Mody SK, Hacker MR, Dodge LE, Thornton K, Schneider B, Haider S. Contraceptive counseling for women who undergo bariatric surgery. J Womens Health (Larchmt). 2011;20(12):1785-1788.

7. Merhi ZO. Revisiting optimal hormonal contraception following bariatric surgery. Contraception. 2013;87(2):131-133.

8. Buchwald H, Avidor Y, Braunwald E, et al. Bariatric surgery: a systematic review and meta-analysis. JAMA. 2004;292(14):1724-1737.

9. Sjostrom L, Peltonen M, Jacobson P, et al. Association of bariatric surgery with long term remission of type 2 diabetes and with microvascular and macrovascular complications. JAMA. 2014;311(22): 2297-2304. 
10. Ponce J, Nguyen NT, Hutter M, Sudan R, Morton JM. American Society for Metabolic and Bariatric Surgery estimation of bariatric surgery procedures in the United States, 2011-2014. Surg Obes Relat Dis. 2015;11(6):1199-1200.

11. Trussell J, Schwarz EB, Guthrie K. Obesity and oral contraceptive pill failure. Contraception. 2009;79(5):334-338.

12. Jain J, Dutton C, Nicosia A, Wajszczuk C, Bode FR, Mishell DR. Pharmacokinetics, ovulation suppression and return to ovulation following a lower dose subcutaneous formulation of Depo-Provera. Contraception. 2004;70(1):11-18.

13. Teal SB, Ginosar DM. Contraception for women with chronic medical conditions. Obstet Gynecol Clin North Am. 2007;34(1):113-126,ix.

14. Jain J, Jakimiuk AJ, Bode FR, Ross D, Kaunitz AM. Contraceptive efficacy and safety of DMPA-SC. Contraception. 2004;70(4):269-275.

15. Kim J, Brethauer S; American Society for Metabolic and Bariatric Surgery Clinical Issues Committee, Position Statement. Metabolic bone changes after bariatric surgery. Surg Obes Relat Dis. 2015;11(2):406-411.

16. Dixon JB, Strauss BJ, Laurie C, O'Brien PE. Changes in body composition with weight loss: obese subjects randomized to surgical and medical programs. Obesity (Silver Spring). 2007;15(5):1187-1198.

17. Coates PS, Fernstrom JD, Fernstrom MH, Schauer PR, Greenspan SL. Gastric bypass surgery for morbid obesity leads to an increase in bone turnover and a decrease in bone mass. J Clin Endocrinol Metab. 2004;89(3):1061-1065.

18. Nogues X, Goday A, Pena MJ, et al. Bone mass loss after sleeve gastrectomy: a prospective comparative study with gastric bypass. Cir Esp. 2010;88(2):103-109.

19. Scibora LM, Ikramuddin S, Buchwald H, Petit MA. Examining the link between bariatric surgery, bone loss, and osteoporosis: a review of bone density studies. Obes Surg. 2012;22(4):654-657.

20. Yu EW. Bone metabolism after bariatric surgery. $J$ Bone Miner Res. 2014;29(7):1507-1518.

21. Westhoff C. Depot-medroxyprogesterone acetate injection (DepoProvera $\left.{ }^{\circledR}\right)$ : a highly effective contraceptive option with proven long term safety. Contraception. 2003;68(2):75-87.

22. Scholes D, Lacroix AZ, Ichikawa LE, Barlow WE, Ott SM. Change in bone mineral density among adolescent women using and discontinuing depot medroxyprogesterone acetate contraception. Arch Pediatr Adolesc Med. 2005;159(2):139-144.

23. Isley M, Kaunitz AM. Update on hormonal contraception and bone density. Rev Endocr Metab Disord. 2011;12(2):93-106.

24. Kaunitz AM, Arias R, McClung M. Bone density recovery after depot medroxyprogesterone acetate injectable contraception use. Contraception. 2008;77(2):67-76.

25. Beckman LM, Beckman TR, Earthman CP. Changes in gastrointestinal hormones and leptin after Roux-en-Y gastric bypass procedure: a review. J Am Diet Assoc. 2010;110(4):571-584.

26. American College of Obstetricians and Gynecologists. ACOG committee opinion No 602: depot medroxyprogesterone acetate and bone effects. Obstet Gynecol. 2014;123(6):1398-1402.

27. Karamanakos SN, Vagenas K, Kalfarentzos F, Alexandrides TK. Weight loss, appetite suppression, and changes in fasting and postprandial ghrelin and peptide-YY levels after Roux-en-Y gastric bypass and sleeve gastrectomy. Ann Surg. 2008;247(3):401-407.

28. Sjöström L. Review of the key results from the Swedish Obese Subjects (SOS) trial - a prospective controlled intervention study of bariatric surgery. J Intern Med. 2013;273(3):219-234.

29. Courcoulas AP, Yanovski SZ, Bonds D, et al. Long-term outcomes of bariatric surgery a National Institutes of Health Symposium. JAMA Surg. 2014;149(12):1323-1329.

30. Maltoni M, Nanni O, Scarpi E, Rossi D, Serra P, Amadori D. High-dose progestins for the treatment of cancer anorexia-cachexia syndrome: a systematic review of randomised clinical trials. Ann Oncol. 2001;12(3): 289-300.
31. Aoyagi T, Terracina KP, Raza A, Matsubara H, Takabe K. Cancer cachexia, mechanism and treatment. World J Gastrointest Oncol. 2015;7(4): 17-29.

32. Bonny AE, Ziegler J, Harvey R, Debanne SM, Secic M, Cromer BA. Weight gain in obese and nonobese adolescent girls initiating depot medroxyprogesterone, oral contraceptive pills, or no hormonal contraceptive method. Arch Pediatr Adolesc Med. 2006;160(1):40-45.

33. Berenson AB, Rahman M. Changes in weight, total fat, percent body fat, and central-toperipheral fat ratio associated with injectable and oralcontraceptive use. Am J Obstet Gynecol. 2009;200(3): 329.e1-329.e8.

34. Mody SK, Han M. Obesity and contraception. Clin Obstet Gynecol. 2014;57(3):501-507.

35. Allman-Farinelli MA. Obesity and venous thrombosis: a review. Semin Thromb Hemost. 2011;37(8):903-907.

36. Curtis KM, Ravi A, Gaffield ML. Progestogen-only contraceptive use in obese women. Contraception. 2009;80(4):346-354.

37. Lopez LM, Edelman A, Chen M, Otterness C, Trussell J, Helmerhorst FM. Progestin only contraceptives: effects on weight. Cochrane Database Syst Rev. 2013;7:CD008815.

38. Modesto W, Silva dos Santos P, Correia VM, Borges L, Bahamondes L. Weight variation in users of depot medroxyprogesterone acetate, the levonorgestrel releasing intrauterine system and a copper intrauterine device for up to ten years of use. Eur J Contracept Reprod Health Care. 2015;20(1):57-63.

39. Larsen TB, Sorensen HT, Gislum M, Johnson SP. Maternal smoking, obesity and risk of venous thromboembolism during pregnancy and the puerperium: a population based nested case-control study. Thromb Res. 2007;120(4):505-509.

40. Murthy AS. Obesity and contraception: emerging issues. Semin Reprod Med. 2010;28(2):156-164.

41. Horvei LD, Braekkan SK, Mathiesen EB, Njolstad I, Wilsgaard T, Hansen JB. Obesity measures and risk of venous thromboembolism and myocardial infarction. Eur J Epidemiol. 2014;29(11):821-830.

42. Yang G, De Staercke C, Hooper WC. The effects of obesity on venous thromboembolism: a review. Open J Prev Med. 2013;2(4):499-509.

43. Mantha S, Karp R, Raghavan V, Terrin N, Bauer KA, Zwicker JI. Assessing the risk of venous thromboembolic events in women taking progestin-only contraception: a meta-analysis. BMJ. 2012;345:e4944

44. Pantoja M, Medeiros T, Baccarin MC, Morais SS, Bahamondes L, Fernandez AM. Variations in body mass index of users of depot-medroxyprogesterone acetate as a contraceptive. Contraception. 2010;81(2): $107-111$.

45. CDC [webpage on the Internet]. The Medical Eligibility Criteria for Contraceptive Use. Centers for Disease Control [cited April 23, 2013]. Available from: http://www.cdc.gov/reproductivehealth/unintendedpregnancy/USMEC.htm. Accessed May 2, 2016.

46. Merki-Feld G, Skouby S, Serfaty D, et al. European society of contraception statement on contraception in obese women. Eur J Contracept Reprod Health Care. 2015;20(1):19-28.

47. Popovic DS, Popovic LS. Obesity and breast cancer - association even more relevant in males? Eur J Intern Med. 2016;29:e11-e12.

48. Prentice RL. Postmenopausal hormone therapy and the risks of coronary heart disease, breast cancer, and stroke. Semin Reprod Med. 2014;32(6):419-425.

49. Kuhl H, Stevenson J. The effect of medroxyprogesterone acetate on estrogen-dependent risks and benefits - an attempt to interpret the Women's Health Initiative results. Gynecol Endocrinol. 2006;22(6):303-317.

50. Li CI, Beaber EF, Chen Tang MT, Porter PL, Daling JR, Malone KE Effect of depo-medroxyprogesterone acetate on breast cancer risk among women 20 to 44 years of age. Cancer Res. 2012;72(8):2028-2035.

51. Byers T, Sedjo RL. Does intentional weight loss reduce cancer risk? Diabetes Obes Metab. 2011;13(12):1063-1072. 


\section{Publish your work in this journal}

Open Access Journal of Contraception is an international, peerreviewed, open access, online journal, publishing original research, reports, reviews and commentaries on all areas of contraception. In addition to clinical research, demographics and health-related aspects, the journal welcomes new findings in animal and preclinical studies relating to understanding the biological mechanisms and practical development of new contraceptive agents. The manuscript management system is completely online and includes a very quick and fair peer-review system. Visit http://www.dovepress.com/testimonials.php to read real quotes from published authors.

Submit your manuscript here: https://www.dovepress.com/open-access-journal-of-contraception-journal 